\title{
Is Cytoreductive Surgery and Hyperthermic Intraperitoneal Chemotherapy a Safe and Effective Procedure for Treating Patients With a Peritoneal Surface Malignancy?
}

\author{
Dong-Guk Park \\ Department of Surgery, Dankook University College of Medicine, Cheonan, Korea
}

See Article on Page 16-22

Multimodality therapy, such as a combination of cytoreductive surgery (CRS) and hyperthermic intraperitoneal chemotherapy (HIPEC), is associated with improved survival for selected patients with peritoneal metastasis of various cancer types. Despite this, this procedure is not widely accepted due to its high rates of morbidity and mortality. In several studies on the combined use of CRS and HIPEC to treat patients with a variety of cancer types, the rates of grade III/IV morbidity and mortality ranged from $22 \%$ to $34 \%$ and from $0.8 \%$ to $4.1 \%$, respectively. Common major postoperative complications included neutropenia, anastomotic leakage, pneumonia, postoperative bleeding, intra-abdominal abscess, systemic sepsis, wound infection, pleural effusion, and renal insufficiency [1].

Kim et al. [2] reported 30-day rates of postoperative morbidity and mortality of $30.4 \%$ and $0 \%$, respectively, which are comparable to the results reported in Newton et al. [1] and Jo et al. [3]. The occurrence of major complications was found to be associated with the number of organ resections (more than 3 ), the duration of surgery ( $\geq 630$ minutes) and the amount of blood loss $(\geq 600$ $\mathrm{mL}$ ) during surgery. However, contrary to other reports, all four patients with recurred colorectal cancer treated with cetuximab had grade III postoperative complications [2].

Recently, we reported the results of a 2-year follow-up at our center. The rate of morbidity above grade III was $22.2 \%$. Pulmonary complications, such as pleural effusion and pneumonia,

Correspondence to: Dong-Guk Park, M.D.

Department of Surgery, Dankook University College of Medicine,

119 Dandae-ro, Dongnam-gu, Cheonan 31116, Korea

Tel: +82-41-550-3931, Fax: +82-41-565-6167

E-mail:dkpark@dankook.ac.kr

(c) 2017 The Korean Society of Coloproctology

This is an open-access article distributed under the terms of the Creative Commons Attribution NonCommercial License (http://creativecommons.org/licenses/by-nc/4.0) which permits unrestricted noncommercial use, distribution, and reproduction in any medium, provided the original work is properly cited. were the most common (29.6\%) complications, followed by acute renal failure (14.8\%), wound problems (13.0\%), and prolonged ileus $(11.1 \%)$. Anastomotic leakage developed in 1 patient. The rate of mortality was $7.4 \%$. The causes of death included pulmonary complication, septic shock related to anastomotic leakage, and hypovolemic shock from gastrointestinal bleeding [3].

According to data from the National Surgical Quality Improvement Program (NSQIP), age $\geq 60$ years, preoperative albumin $<3$ $\mathrm{g} / \mathrm{dL}$, and poor preoperative performance status are major factors contributing to morbidity [4]. Major operative factors are the peritoneal cancer index, bowel resection, diaphragmatic involvement [5], the use of a distal pancreatectomy, and surgeon's experience. Furthermore, hepatobiliary procedures, urologic procedures, and preoperative use of bevacizumab have weak associations with the occurrence of morbidity. In a NSQIP analysis, increased operative time, intraoperative transfusion, and the use of a gastrectomy with intraperitoneal chemotherapy were associated with increased morbidity and mortality [4]. Studies suggest that perioperative systemic chemotherapy does not appear to increase the rate of major morbidities from the use of a combined CRS and HIPEC procedure, with the possible exception of preoperative bevacizumab, which has been associated with increased operative complications. Intraperitoneal chemotherapy increases neither morbidity nor mortality greatly over surgery alone [4].

One of the major limitations of this study [2] is that the number of patients treated with the combined CRS and HIPEC procedure was small $(n=23)$. Some studies suggest that approximately 140 180 procedures are needed to minimize severe morbidity [6], but in this paper, even though the number of patients was small, the rate of morbidity was comparable to the value in a report published by a large-volume center. This suggests that the combined use of CRS and HIPEC will become a somewhat popular procedure, in spite of its being a complex procedure. The combined use of CRS and HIPEC to treat patients with disseminated intra-abdominal malignancies is a complex procedure with the potential for high morbidity and mortality. However, when the combined CRS and HIPEC procedure is performed by experienced sur- 


\section{Coloproctology Dong-Guk Park}

geons at high-volume centers, it can be associated with long-term survival with acceptable morbidity and mortality rates. Because serious postoperative complications are associated with poor quality of life, the selection of patients to undergo this combined procedure is very important. Preoperatively, a discussion of all factors contributing to CRS and HIPEC morbidity and mortality by the members of a multidisciplinary committee is mandatory.

\section{CONFLICT OF INTEREST}

No potential conflict of interest relevant to this article was reported.

\section{REFERENCES}

1. Newton AD, Bartlett EK, Karakousis GC. Cytoreductive surgery and hyperthermic intraperitoneal chemotherapy: a review of factors contributing to morbidity and mortality. J Gastrointest Oncol 2016;7:99-111.
2. Kim WR, Hur H, Min BS, Baik SH, Lee KY, Kim NK. Single center experience with hyperthermic intraperitoneal chemotherapy. Ann Coloproctol 2017;33:16-22.

3. Jo MH, Suh JW, Yun JS, Namgung H, Park DG. Cytoreductive surgery and intraperitoneal chemotherapy for peritoneal carcinomatosis of colorectal cancer: 2-year follow-up results at a single institution in Korea. Ann Surg Treat Res 2016;91:157-64.

4. Bartlett EK, Meise C, Roses RE, Fraker DL, Kelz RR, Karakousis GC. Morbidity and mortality of cytoreduction with intraperitoneal chemotherapy: outcomes from the ACS NSQIP database. Ann Surg Oncol 2014;21:1494-500.

5. Franssen B, Tabrizian P, Weinberg A, Romanoff A, Tuvin D, Labow D, et al. Outcome of cytoreductive surgery and hyperthermic intraperitoneal chemotherapy on patients with diaphragmatic involvement. Ann Surg Oncol 2015;22:1639-44.

6. Polanco PM, Ding Y, Knox JM, Ramalingam L, Jones H, Hogg $\mathrm{ME}$, et al. Institutional learning curve of cytoreductive surgery and hyperthermic intraperitoneal chemoperfusion for peritoneal malignancies. Ann Surg Oncol 2015;22:1673-9. 